\title{
Biosynthesis of Anisotropic Silver Nanoparticles by Bhargavaea indica and Their Synergistic Effect with Antibiotics against Pathogenic Microorganisms
}

\author{
Priyanka Singh,, Yeon Ju Kim, ${ }^{1}$ Hina Singh,, Ramya Mathiyalagan, \\ Chao Wang, ${ }^{1}$ and Deok Chun Yang ${ }^{1,2}$ \\ ${ }^{1}$ Department of Oriental Medicinal Biotechnology, College of Life Sciences, Kyung Hee University, Republic of Korea \\ ${ }^{2}$ Graduate School of Biotechnology and Ginseng Bank, College of Life Sciences, Kyung Hee University, \\ Yongin 446-701, Republic of Korea \\ Correspondence should be addressed to Yeon Ju Kim; yeonjukim@khu.ac.kr and Deok Chun Yang; dcyang@khu.ac.kr
}

Received 18 November 2014; Accepted 1 January 2015

Academic Editor: An-Ya Lo

Copyright (c) 2015 Priyanka Singh et al. This is an open access article distributed under the Creative Commons Attribution License, which permits unrestricted use, distribution, and reproduction in any medium, provided the original work is properly cited.

\begin{abstract}
The strain Bhargavaea indica DC1 isolated from four-year-old P. ginseng rhizospheric soil was used to perform rapid and extracellular biosynthesis of anisotropic silver nanoparticles. The ultraviolet-visible (UV-vis) spectra of the reaction mixture containing silver nanoparticles showed a peak at $460 \mathrm{~nm}$, corresponding to the surface plasmon absorbance of silver nanoparticles. Field-emission transmission electron microscopy (FE-TEM) structural characterization revealed the nanobar, pentagon, spherical, icosahedron, hexagonal, truncated triangle, and triangular nanoparticles, with the size range from 30 to $100 \mathrm{~nm}$. The energydispersive X-ray (EDX) analysis and elemental mapping results also confirmed that the silver was the predominant component of isolated nanoparticles. The X-ray diffraction (XRD) results correspond to the purity of silver nanoparticles and dynamic light scattering (DLS) result indicated that the average diameter of particles was $111.6 \mathrm{~nm}$. In addition, enhancement in antimicrobial activity of commercial antibiotics was observed against various pathogenic microorganisms such as Vibrio parahaemolyticus, Salmonella enterica, Staphylococcus aureus, Bacillus anthracis, Bacillus cereus, Escherichia coli, and Candida albicans.
\end{abstract}

\section{Introduction}

Nanomaterials have been studied intensively and have received considerable attention in various scientific fields due to unique properties including ultra-small size, high surfacearea-to-mass ratio, and high reactivity [1]. Chemical and physical methods for the synthesis of metal nanoparticles are well known, but the methodologies are expensive and not environmentally friendly, thus limiting the applications of metal nanoparticles in biological and medical platforms [2]. To overcome the limitation of physiochemical methodologies, the simple, low-cost, and eco-friendly technologies are needed whereby these nanoparticles can be synthesized while avoiding the use of toxic chemicals and solvents. The green synthesis is a feasible alternative, and various biological species have been well studied for this purpose, including bacteria, yeast, fungi, and plant extracts [2-6]. Such research has led to new product developments and applications of biological species in different fields [7].

Among the many metal nanoparticles, silver nanoparticles are one of the most studied and prominent nanoproducts. Silver nanoparticles are well known for their inhibitory and bactericidal effects and have a broad range of applications in many fields $[8,9]$. The reduction of $\mathrm{Ag}^{+}$ions by some biological species and products causes the synthesis of silver nanoparticles with enhancing properties due to an increase in the surface plasmon resonance and large effective scattering cross section of individual nanoparticles [10]. Moreover, the activity of metal nanoparticles at nanostructure level can be easily tuned by varying specific characteristics [11]. These 
unique properties of nanoparticles make them a superior and indispensable choice in many areas of biological activity [12].

Anisotropic particles provide greater versatility and options in many cases and applications. Studies suggest that shape of particles is one of the important factors, as most of the intrinsic properties depend on it. Nonspherical nanoparticles such as pentagons, cubes, nanospheres, icosahedrons, hexagons, truncated triangles, triangular prisms, and other more exotic structures of metal nanoparticles exhibit unique and fine-tuned properties which strongly differ and are more pronounced from those of spherical nanoparticles. Their unusual physiochemical property makes them ideal structures and valuable choice to be used for biomedical labelling, optical sensing and imaging, photonics, electronics, catalysis, and electronic devices among others. Furthermore, anisotropic particles provide templates for further synthesis of novel nanomaterials [9, 14]. Anisotropic silver and gold nanoparticles synthesized by chemical, physical, and biological system have been studied and demonstrated for their importance and applications in medical field [15-17].

The antibiotic resistance mechanism in microorganisms has become a major concern and has received considerable attention in the medical field [18]. Silver nanoparticles can play a major role in this area, as they have been found to be active against many multidrug resistant pathogenic microorganisms [19] and in addition, most of the microorganisms are less likely to develop defense mechanisms against metals as compared to antibiotics. Thus, here we report the biosynthesis of anisotropic silver nanoparticles by bacterial strain isolated from four-year-old Panax ginseng rhizospheric soil.

P. ginseng is a slow-growing, perennial, and herbal medicinal plant. The rhizospheric soil microflora of the P. ginseng have been well studied and explored for various applications such as the bioconversion of ginsenosides [20], plant growth promoting activity [21], and antimicrobial activity [22]. In the present study, a bacterial strain isolated from $P$. ginseng rhizospheric soil is demonstrated to be useful for an application in the field of nanotechnology, that is, for the synthesis of anisotropic silver nanoparticles. The biosynthesised anisotropic silver nanoparticles were characterized by UV-vis, FE-TEM, EDX, elemental mapping, XRD, and DLS. In addition, nanoparticles were tested for their antimicrobial properties against pathogenic microorganisms including Bacillus anthracis, Vibrio parahaemolyticus, Salmonella enterica, Staphylococcus aureus, Escherichia coli, Bacillus cereus, and Candida albicans. Furthermore, the silver nanoparticles have been tested in combination with commercial antibiotics including lincomycin, oleandomycin, novobiocin, vancomycin, penicillin $\mathrm{G}$, rifampicin, and cycloheximide, for their ability to enhance the antibiotics' antimicrobial effects against pathogenic microorganisms.

\section{Experimental}

2.1. Media, Chemicals, and Microorganism. All media used were purchased from Difco, MB cell, Seoul, Korea. Analytical-grade silver nitrate $\left(\mathrm{AgNO}_{3}\right)$ and cycloheximide were purchased from Sigma-Aldrich Chemicals, USA. All antibiotics used were purchased from Oxoid Ltd., England: vancomycin (VA30) $30 \mu \mathrm{g} /$ disk, rifampicin (RD5) $5 \mu \mathrm{g} / \mathrm{disk}$, oleandomycin (OL15) $15 \mu \mathrm{g} /$ disk, penicillin G (P10) $10 \mu \mathrm{g} / \mathrm{disk}$, novobiocin (NV30) $30 \mu \mathrm{g} / \mathrm{disk}$, and lincomycin (MY15) $15 \mu \mathrm{g} /$ disk.

The pathogenic bacterial strains used Bacillus anthracis [NCTC 10340], Vibrio parahaemolyticus [ATCC 33844], Salmonella enterica [ATCC 13076], Staphylococcus aureus [ATCC 6538], Escherichia coli [ATCC 10798], Bacillus cereus [ATCC 14579], and Candida albicans [KACC 30062]. The bacterial strains were cultured on nutrient agar media at $37^{\circ} \mathrm{C}$ and preserved at $-70^{\circ} \mathrm{C}$ in glycerol stock vials for further study. C. albicans was cultured on Sabouraud dextrose agar at $28^{\circ} \mathrm{C}$ and preserved at $-70^{\circ} \mathrm{C}$ in glucose yeast peptone broth (GYP) glycerol stocks vials.

2.2. Isolation of Bacteria. A bacterial strain $\mathrm{DCl}$ was isolated from four-year-old $P$. ginseng rhizospheric soil (Sodang, Republic of Korea) on tryptic soy agar (TSA) and incubated at $30^{\circ} \mathrm{C}$ for $24 \mathrm{~h}$. Its genomic DNA was extracted using a commercial genomic DNA extraction kit (Core Bio System). The 16S rRNA gene was amplified from the chromosomal DNA of the isolated strain using the universal bacterial primer set 27F, 518F, 800R, and 1512R [23, 24]. The purified PCR products were sequenced by Genotech (Daejeon, Korea). The $16 \mathrm{~S}$ rRNA gene sequences of related taxa were obtained from the GenBank database and EzTaxon-e server [25].

2.3. Extracellular Synthesis of Silver Nanoparticles. The biological synthesis of silver nanoparticles was carried out as reported [11] with slight modification. Briefly, the bacteria were grown in tryptic soy broth (TSB) and kept in incubation for $24 \mathrm{~h}$ in a shaker held at $37^{\circ} \mathrm{C}$ and $120 \mathrm{rpm}$. The culture was then centrifuged at $8000 \mathrm{rpm}$ for $5 \mathrm{~min}$ and the supernatant was used for the synthesis of silver nanoparticles. $\mathrm{AgNO}_{3}$ was added to the culture supernatant to reach the concentration of $1 \mathrm{mM}$, and this reaction mixture was incubated in the orbital shaker at $200 \mathrm{rpm}$ and $25^{\circ} \mathrm{C}$. The extracellular synthesis of silver nanoparticles was monitored by visual inspection for a change in the color of the culture medium. After incubation was complete, the reaction mixture was first centrifuged at $2000 \mathrm{rpm}$ for $5 \mathrm{~min}$ to remove any medium components, and then the biosynthesised silver nanoparticles were collected by high-speed centrifugation at $16000 \mathrm{rpm}$ for $20 \mathrm{~min}$. The product obtained was washed several times by centrifugation and redispersion in water to remove the unconverted silver ions and any medium components. Finally, the silver nanoparticles were collected in the form of a pellet and used for characterization.

2.4. Characterization of Silver Nanoparticles. UV-vis, FETEM, EDX, elemental mapping (quantitation method: CliffLorimer ratio), and DLS were used to characterize the size, shape, composition, and other properties of the silver nanoparticles. The bioreduction was monitored by using a UV-vis spectrophotometer (Ultrospec 2100 pro); this was carried out by scanning the absorbance spectra of the reaction mixture over the wavelength range of $300-700 \mathrm{~nm}$. TEM, 
EDX, and elemental mapping were performed by using a FE-TEM (JEM-2100F, JEOL) operated at $200 \mathrm{kV}$. Samples for FE-TEM, EDX, and elemental mapping were prepared by dropping the unstained silver nanoparticles pellet solution onto a carbon-coated copper grid and drying in an oven at $60^{\circ} \mathrm{C}$. FE-TEM was used for structural characterization of nanoparticles. EDX and elemental mapping were used to study the distribution of elements. The X-ray diffraction (XRD) analyses were performed on X-ray diffractometer, D8 Advance, Bruker, Germany, operated at $40 \mathrm{kv}, 40 \mathrm{~mA}$, with $\mathrm{CuK} \alpha$ radiation, at a scanning rate of $6^{\circ} / \mathrm{min}$, step size 0.02 , over the $2 \theta$ range of $20-80^{\circ}$. The silver nanoparticles have been collected by centrifugation and washing several times with sterile water. Then, the particles were finally recovered by air drying and obtained in powder form.

DLS (particles size analyzer, Photal, Otsuka Electronics, Japan) was used to monitor size distribution profile of biologically synthesized silver nanoparticles. The hydrodynamic diameters and polydispersity index (PDI) were analysed at $25^{\circ} \mathrm{C}$. As a reference dispersive medium pure water with refractive index 1.3328 , viscosity 0.8878 , and dielectric constant 78.3 was used.

The stability of silver nanoparticles was observed by keeping the anisotropic silver nanoparticles reaction mixture for different time interval at room temperature. In addition, the effect of change in $\mathrm{pH}$ on the stability of the silver nanoparticles was studied. The sodium hydroxide solution was added in the range of $4-10 \mathrm{pH}$ and then the solution has been scanned by UV-vis spectrophotometer to observe the absorbance.

2.5. Analysis of Antimicrobial Activity of Silver Nanoparticles. Antimicrobial activity of the silver nanoparticles against pathogenic microorganisms, S. aureus, B. anthracis, V. parahaemolyticus, S. enterica, E. coli, B. cereus, and C. albicans, was tested by the well diffusion method, carried out using MullerHinton agar (MHA) plates. An overnight log-phase culture of each strain was spread evenly on an MHA plate by using a glass spreader. Wells were made on the MHA plates by using a gel puncture. $50 \mu \mathrm{L}$ of the biosynthesised silver nanoparticles reaction mixture was added into each of four wells and the plates were incubated at $37^{\circ} \mathrm{C}$ for $24 \mathrm{~h}$.

Simultaneously, the synergistic effects of different commercial antibiotics with silver nanoparticles were also tested by the disk diffusion method. Similarly, an overnight logphase culture of each strain was spread evenly on an MHA plate. Then, antibiotic disk of each of the commercial antibiotics was placed on the plate, and $30 \mu \mathrm{L}$ of a $100 \mathrm{ppm}$ solution of silver nanoparticles was added over each disk $(10 \mu \mathrm{L}, 3$ times). Antibiotic disks without silver nanoparticles were used as negative control. The plates were allowed to dry and then incubated at $37^{\circ} \mathrm{C}$ for $24 \mathrm{~h}$. After incubation, the susceptibility pattern of the test organisms was determined by measuring the diameter of the zone of inhibition for both the well diffusion and disk diffusion methods. Similarly, cycloheximide antimicrobial activity against $C$. albicans was evaluated and compared with addition of silver nanoparticles with same concentration.

\section{Results and Discussion}

3.1. Isolation and Identification of Bacteria. The bacterial strain DC1 used herein to carry out the extracellular biosynthesis of silver nanoparticles was isolated from four-year-old P. ginseng rhizospheric soil and showed $99.79 \%$ similarity with Bhargavaea indica [26]. The strain Bhargavaea indica DC1 $16 \mathrm{~S}$ rRNA sequence has been submitted to NCBI with accession number KM819013. The strain has been deposited to KCTC culture collection (KCTC 33595).

3.2. Synthesis and Characterization of Silver Nanoparticles. In this study, silver nanoparticles were successfully synthesised extracellularly in the culture supernatant of $B$. indica DC1.

The first indication of silver nanoparticles synthesis was that, upon addition of $1 \mathrm{mM}$ silver nitrate into the flask containing a $24 \mathrm{~h}$ culture supernatant of the strain, the medium gradually turned brown, whereas no color change was observed in the control without bacteria. This brown color could be due to the excitation of surface plasmon vibrations, which would arise from the formation of silver nanoparticles in the reaction mixture [11]. Interestingly, the culture supernatant incubated with $1 \mathrm{mM}$ silver nitrate mediated the biosynthesis of silver nanoparticles within $48 \mathrm{~h}$. The $1 \mathrm{mM}$ concentration for the synthesis of silver nanoparticles was selected based on the silver nitrate tolerating capacity of the isolate $B$. indica DCl. The strain showed optimum growth at $1 \mathrm{mM}$ concentration. Moreover, the concentration of precursor $(0.1-1 \mathrm{mM})$ for the synthesis of nanoparticles does not show any effect on the end product of reaction; thus the shape and size of nanoparticles were not affected by the precursor concentration ranging.

UV-vis is the most established method for structural characterization of metal nanoparticles. The reduction of silver ions during exposure to the supernatant of the strain DC1 was easily monitored by UV-vis. After the incubation period, the reaction mixture was scanned in the range of $300-700 \mathrm{~nm}$, showing a major absorbance peak at $460 \mathrm{~nm}$ (Figure 1(a)). The broad peak is due to the anisotropic nature of the particles. It has been reported that the band in this region corresponds to the surface plasmon resonance of silver nanoparticles [27]. Thus, the reaction mixture indicates the formation of silver nanoparticles. The nanoparticles synthesize extracellularly, avoiding the downstream processing needed for intracellular synthesis.

Various researchers have reported evidence of the extracellular biosynthesis of silver nanoparticles, including TEM structural characterizations [28]. The anisotropic silver nanoparticles synthesised were successfully characterized by FE-TEM and revealed that the particles ranged in size from 30 to $100 \mathrm{~nm}$ and were of various shapes including nanobar, pentagon, spherical, icosahedron, hexagonal, truncated triangle, and triangular shapes (Figure 1(b)). Although the monodispersity is the main issue associated with the nanoparticles synthesis, the biosynthesised anisotropic silver nanoparticles are of great advantage in biological platforms, since the silver nanoparticles undergo shape-dependent interactions with microorganisms [9]. Reports suggest that the biosynthesised silver nanoparticles of different shapes can be separated by 


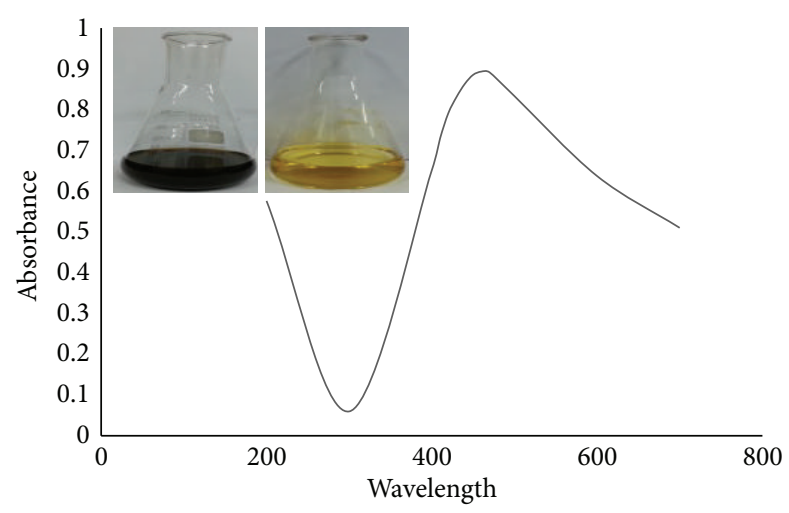

(a)

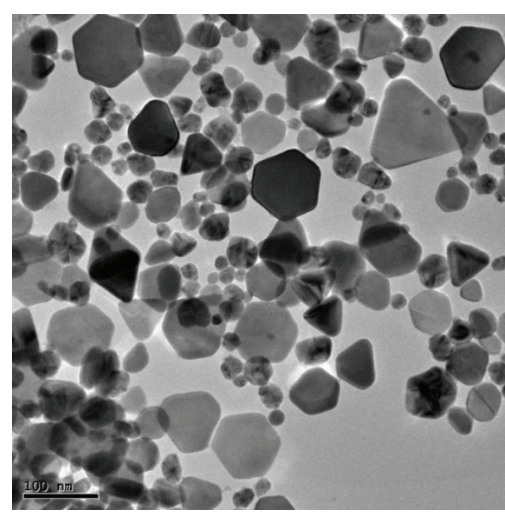

(b)

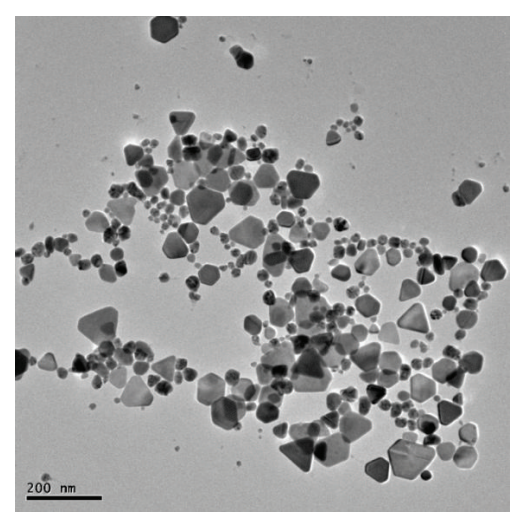

(c)

FIGURE 1: UV-vis spectra of culture supernatant of Bhargavaea indica DC1 treated with (1 mM) $\mathrm{AgNO}_{3}$ (a). Transmission electron micrograph of anisotropic silver nanoparticles synthesised by Bhargavaea indica DC1, 100 and $200 \mathrm{~nm}$ (b).

centrifugation, as the nanoparticles show shape-dependent sedimentation behaviour [29]. The biological synthesis of anisotropic gold and silver nanoparticles by Aspergillus niger has been reported previously. The syntheses of anisotropic silver nanoparticles by Morganella psychrotolerans [30] and anisotropic gold nanoparticles by the fungal strain Rhizopus oryzae [31] have been studied. To our knowledge, this is the first report of the ability of $B$. indica DC1 to synthesise silver nanoparticles of various shapes and sizes. The exact mechanism behind the synthesis remains to be elucidated, but based on reports, the enzyme and proteins synthesised extracellularly by bacterial strain in the reaction mixture help in reducing the silver nitrate to silver nanoparticles. Moreover, the particles synthesised without any additional stabilizing and capping agent thus eliminating the main step of chemical synthesis and overcome the limitations. The cell-free supernatant containing extracellular enzymes and proteins functions as a reducing, shape-directing, and stabilizing agent $[2,31]$.

The purity of the biosynthesised silver nanoparticles was examined by EDX (Figure 2(a)); the EDX spectrum displayed an optical absorption band peak at $3 \mathrm{keV}$, which is the characteristic peak of nanosized metallic silver, corresponding to surface plasmon resonance [32]. Carbon and copper signals originating in the low-energy part of the spectrum arose from the use of the carbon-coated copper TEM grid. Similar results have been observed in the study of biosynthesised silver nanoparticles by Streptomyces hygroscopicus [33] and mangrove Streptomyces sp. BDUKAS10 [6].

Figure 2(b) shows an elemental mapping result for the biosynthesised silver nanoparticles, showing the relative locations of elements in the TEM cross section. Results clearly demonstrate that the maximum deposition corresponds to silver nanoparticles, 55.95\%. Other elements observed included carbon, $12.65 \%$, and copper, $28.68 \%$; these two elements were observed due to the carbon-coated copper grid used for TEM structural analysis. Chlorine was also observed in a very low amount, $2.71 \%$, in the cross sections of the nanoparticles themselves. The distribution of silver element was clearly visible in elemental maps and was the predominant element. Thus, the EDX spectrum and elemental mapping gave confirmation that the nanoparticles consisted of silver only [6]. Figure 3 showed the X-ray diffraction pattern of silver nanoparticles and exhibited intense peaks in the whole spectrum of $2 \theta$ value ranging from 20 to 80 and this pattern was similar to Bragg's reflection of silver nanocrystals.

The DLS measurements of number, intensity, and volume distribution of nanoparticles show the size range from 50 to $150 \mathrm{~nm}$ with an average diameter of $111 \mathrm{~nm}$ and PDI 0.233 (Figures 4(a)-4(c)) which is different from the size measured by TEM, that is, $30-100 \mathrm{~nm}$. Reports suggest that the size difference between the particles size analyses by DLS and 


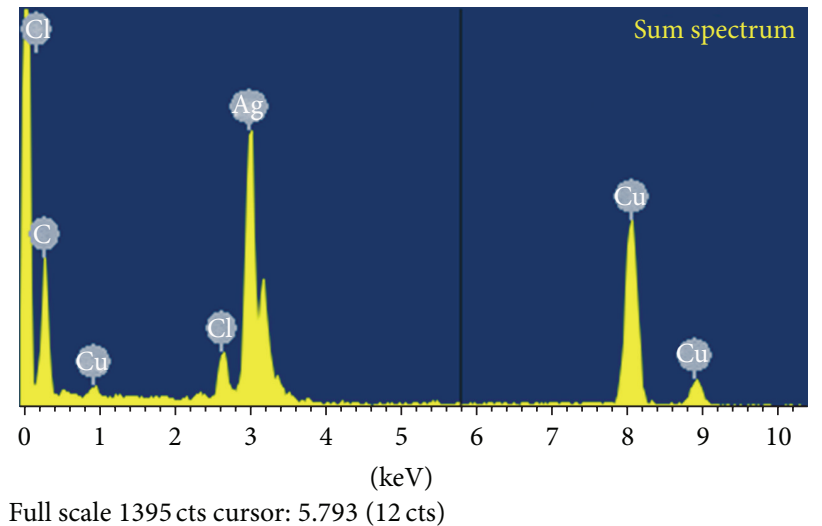

(a)

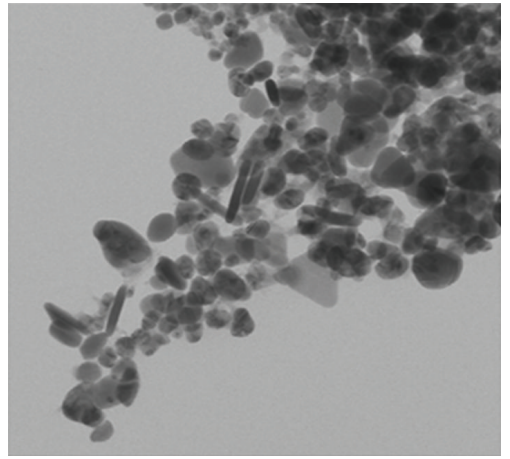

Electron image 1

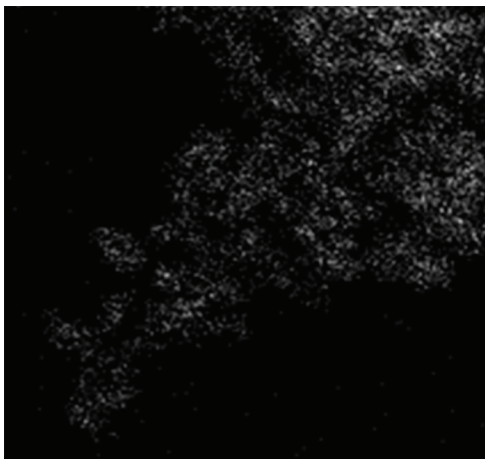

Ag Lal

(b)

FIGURE 2: EDX spectra of the whole scan area showing major peak of anisotropic silver nanoparticles at $3 \mathrm{keV}$ (a) and TEM micrograph of anisotropic silver nanoparticles pellet solution and silver nanoparticles; $55.95 \%$ (b), respectively.

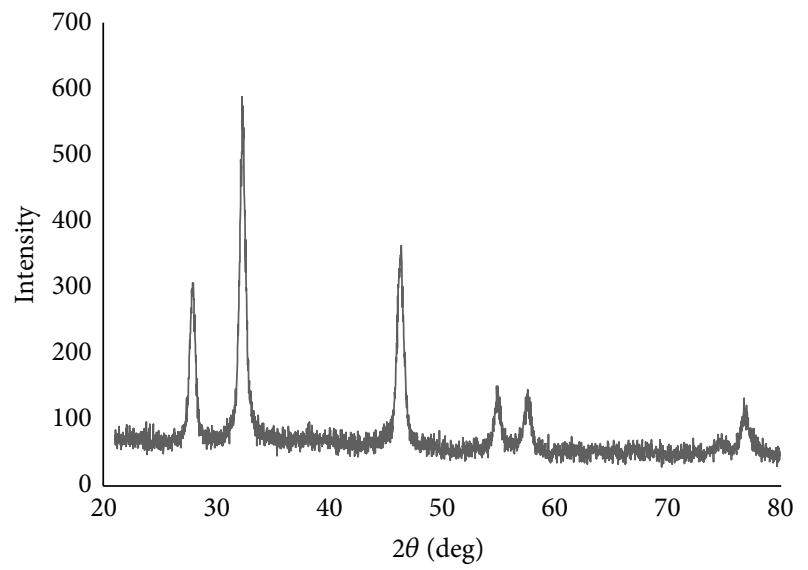

FIGURE 3: X-ray diffraction patterns of anisotropic silver nanoparticles obtained by Bhargavaea indica DC1.

TEM measurement has difference due to the fact that TEM visualizes the metallic core of the particles, whereas DLS measures the hydrodynamic diameter of the particles [34]. Thus, $B$. indica $\mathrm{DC1}$ was demonstrated to be suitable for the simple, green, nonhazardous, economical, and eco-friendly synthesis of antimicrobial anisotropic silver nanoparticles without any additional reducing and stabilizing agent.

Based on the incubation of reaction mixture for different time interval at room temperature, the stability of the nanoparticles was observed and was found that there was no observable variation in the UV-vis spectrum, which directed the stable nature of the anisotropic silver nanoparticles. In addition, the silver nanoparticles solution was observed before and after the addition of sodium hydroxide. In results, no major change in the wavelength was observed, which further confirmed the stability.

3.3. Antimicrobial Activity of Silver Nanoparticles against Test Microorganisms. The biosynthesised silver nanoparticles displayed antimicrobial activity against a range of pathogenic microorganisms such as $C$. albicans, $V$. parahaemolyticus, $S$. aureus, B. anthracis, B. cereus, S. enterica, and E. coli (Figure 5). The mean diameter of the zone of inhibition of all the four wells was determined for each microorganism, showing that the silver nanoparticles had the greatest antimicrobial activity against $C$. albicans, followed by $V$. parahaemolyticus, $S$. aureus, B. anthracis, B. cereus, S. enterica, and then E. coli. The study has been done in duplicate and average results were interpreted in Table 1 . The results clearly indicate that 


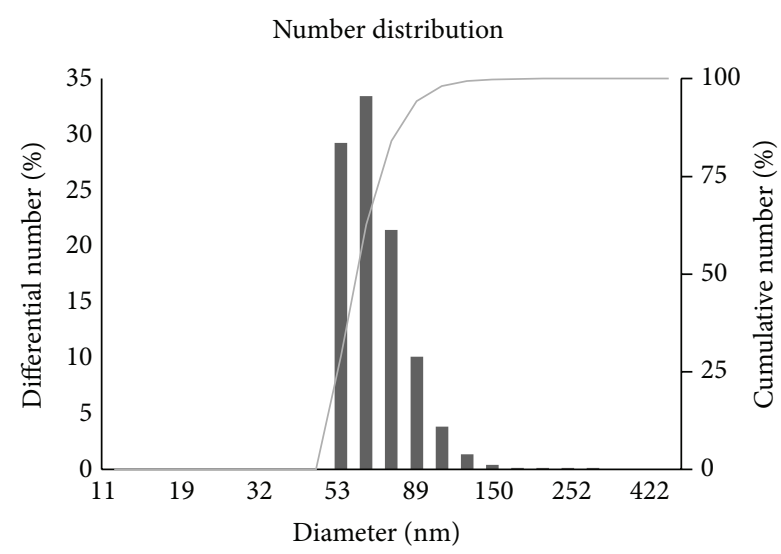

(a)

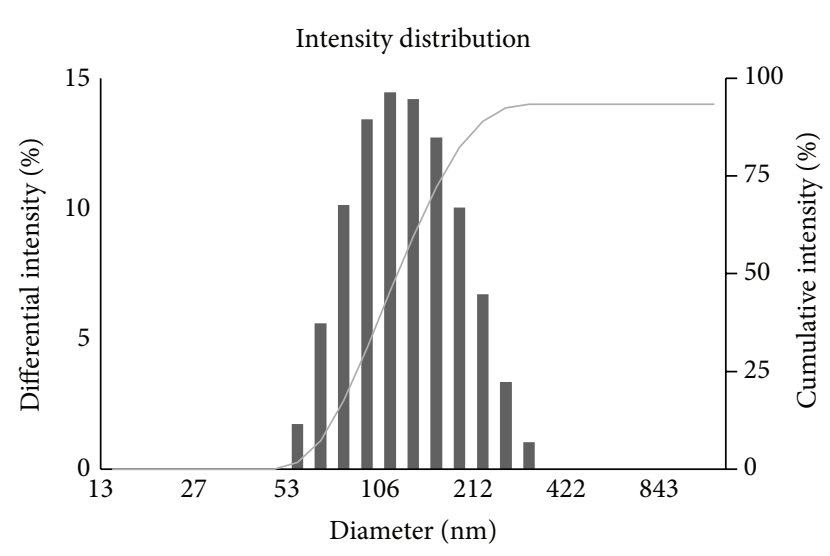

(b)

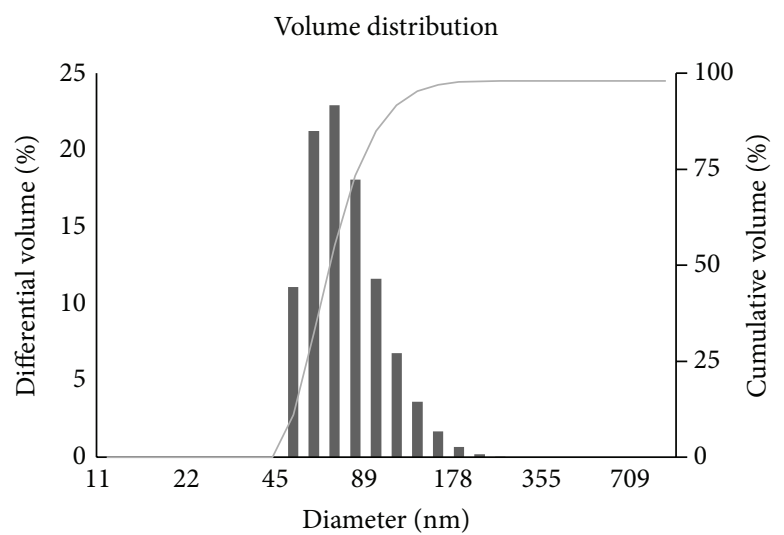

(c)

FIGURE 4: Particles size distribution of anisotropic silver nanoparticles according to number (a), intensity (b), and volume (c), respectively.

silver nanoparticles exert antimicrobial activity against tested microorganisms. Many studies have reported the antimicrobial activity of silver nanoparticles against pathogenic microorganisms $[7,11]$. However, the mechanism of the metal nanoparticles' inhibitory action is still not clearly understood. There are reports suggesting that (i) the electrostatic attraction between the negatively charged bacterial cell and the positively charged nanoparticles leads to accumulation of nanoparticles on the bacterial membrane, resulting in an increase in cell permeability, (ii) the association of silver with oxygen and its reaction with sulfhydryl $(\mathrm{S}-\mathrm{H})$ groups on the cell wall forms R-S-S-R bonds, thereby blocking respiration, (iii) the silver nanoparticles interact with bacterial proteins, which finally leads to cell death, and (iv) the nanoparticles release silver ion which provide additional bactericidal effect [35].

The combination of silver nanoparticles with different commercial antibiotics was evaluated to determine its activity against six pathogenic bacteria using the disk diffusion method. Figure 6(a) represents the antimicrobial activity of partially purified silver nanoparticles and standard antibiotics antimicrobial activity against $S$. enterica, E. coli, V. parahaemolyticus, B. anthracis, B. cereus, and $S$. aureus, respectively. The synergistic effect of antibiotics
TABLE 1: Diameter of zone of inhibition $(\mathrm{mm})$ of reaction mixture $(50 \mu \mathrm{L})$ containing anisotropic silver nanoparticles against pathogenic microorganisms.

\begin{tabular}{lcc}
\hline Number & Pathogenic microorganism & $\begin{array}{c}\text { Zone of } \\
\text { inhibition }(\mathrm{mm})^{\dagger}\end{array}$ \\
\hline 1 & Candida albicans [KACC 30062] & $23 \pm 0.5$ \\
2 & Vibrio parahaemolyticus [ATCC 33844] & $22 \pm 1$ \\
3 & Staphylococcus aureus [ATCC 6538] & $18 \pm 0.8$ \\
4 & Bacillus anthracis [NCTC 10340] & $16 \pm 0.6$ \\
5 & Bacillus cereus [ATCC 14579] & $15 \pm 0.4$ \\
6 & Salmonella enterica [ATCC 13076] & $14 \pm 1$ \\
7 & Escherichia coli [ATCC 10798] & $10 \pm 1$ \\
\hline
\end{tabular}

$\overline{{ }^{\dagger} \text { Mean diameter of four wells, } 50 \mu \mathrm{L} \text { reaction mixture containing anisotropic }}$ silver nanoparticles.

(lincomycin, oleandomycin, novobiocin, vancomycin, penicillin G, and rifampicin) with biosynthesised silver nanoparticles increased the susceptibility of the tested microorganisms (Figure 6(b)). The disk diffusion tests clearly indicated that the strains of S. enterica, E. coli, and V. parahaemolyticus were completely resistant to the antibiotics, whereas the bacterial strains showed sensitivity to the disks when anisotropic 

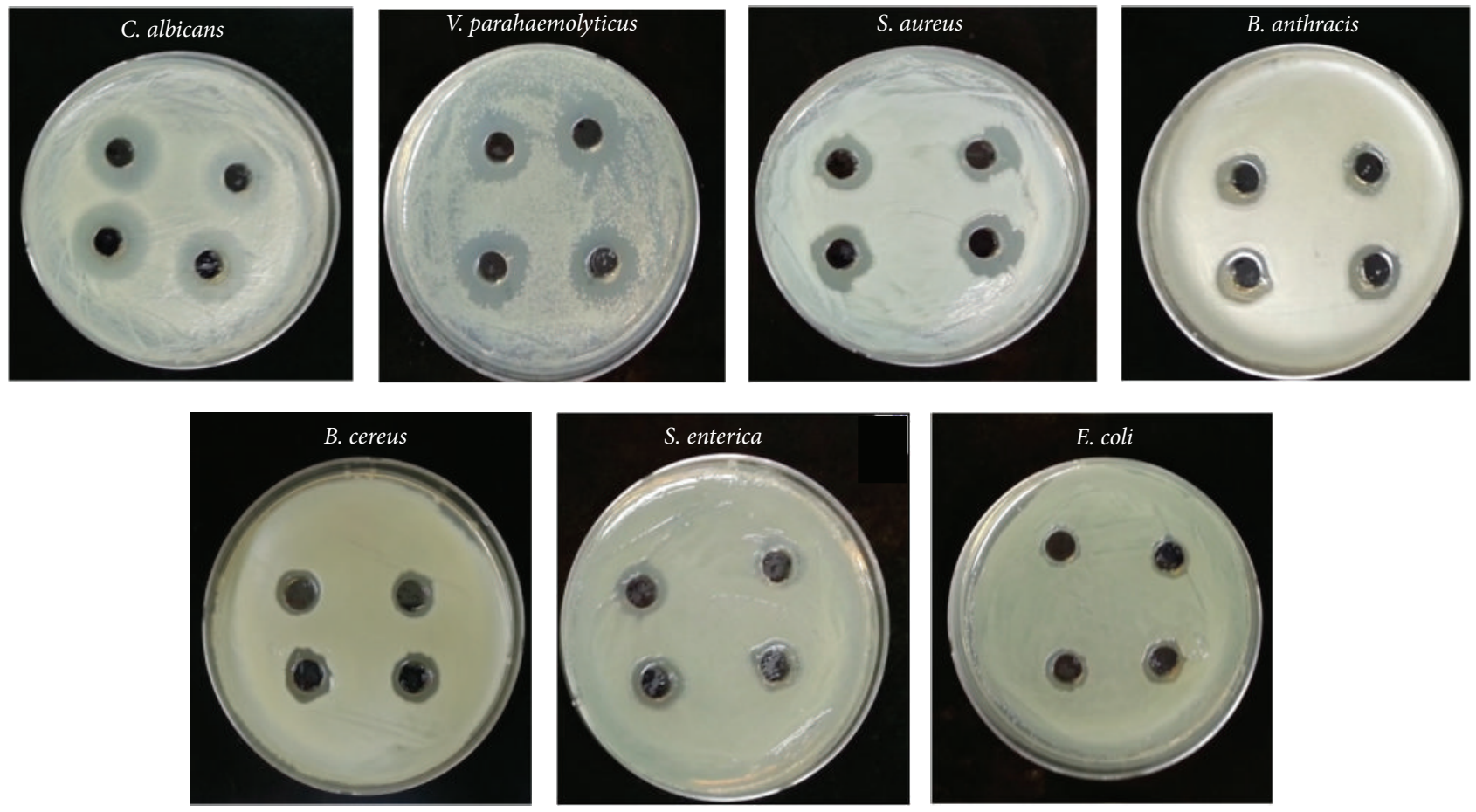

FIGURE 5: Zones of inhibition of $50 \mu \mathrm{L}$ of reaction mixture containing anisotropic silver nanoparticles against pathogenic strains including Candida albicans, Vibrio parahaemolyticus, Staphylococcus aureus, Bacillus anthracis, Bacillus cereus, Salmonella enterica, and Escherichia coli, respectively.

TABLE 2: Individual and combined efficacy of commercial antibiotics with biosynthesised anisotropic silver nanoparticles against pathogenic microorganisms.

\begin{tabular}{|c|c|c|c|c|c|c|c|c|c|}
\hline Pathogenic strain & $\begin{array}{c}\text { MY15 } \\
\text { (a) }\end{array}$ & $\begin{array}{c}\text { MY15 + AgNPs } \\
(b)\end{array}$ & $\mathrm{FA}^{\ddagger}$ & $\begin{array}{l}\text { OL15 } \\
(a)\end{array}$ & $\begin{array}{c}\text { OL15 + AgNPs } \\
(b)\end{array}$ & $\mathrm{FA}^{\neq}$ & $\begin{array}{l}\text { NV30 } \\
\text { (a) }\end{array}$ & $\begin{array}{c}\text { NV30 + AgNPs } \\
(b)\end{array}$ & $\mathrm{FA}^{\neq}$ \\
\hline S. enterica & $0^{\dagger}$ & $12 \pm 0.5$ & 3 & $0^{\dagger}$ & $12 \pm 0.2$ & 3 & $0^{\dagger}$ & $14 \pm 0.9$ & 4.44 \\
\hline E. coli & $0^{\dagger}$ & $13 \pm 0.3$ & 3.69 & $0^{\dagger}$ & $13 \pm 0.6$ & 3.69 & $0^{\dagger}$ & $14 \pm 1$ & 4.44 \\
\hline V. parahaemolyticus & $0^{\dagger}$ & $15 \pm 0.4$ & 5.25 & $0^{\dagger}$ & $15 \pm 0.3$ & 5.25 & $0^{\dagger}$ & $17 \pm 0.6$ & 7.02 \\
\hline Average & & & 3.98 & & & 3.98 & & & 5.3 \\
\hline B. anthracis & $14 \pm 0.1$ & $16 \pm 0.1$ & 0.31 & $15 \pm 0.4$ & $18 \pm 0.2$ & 0.44 & $19 \pm 0.2$ & $22 \pm 0.3$ & 0.34 \\
\hline B. cereus & $16 \pm 0.2$ & $18 \pm 0.6$ & 0.26 & $22 \pm 0.3$ & $24 \pm 0.5$ & 0.19 & $22 \pm 0.1$ & $25 \pm 0.4$ & 0.29 \\
\hline S. aureus & $25 \pm 0.1$ & $28 \pm 0.5$ & 0.25 & $22 \pm 0.2$ & $25 \pm 0.7$ & 0.29 & $30 \pm 0.3$ & $34 \pm 1$ & 0.28 \\
\hline Average & & & 0.27 & & & 0.31 & & & 0.30 \\
\hline Pathogenic strain & $\begin{array}{l}\text { VA30 } \\
(a)\end{array}$ & $\begin{array}{c}\text { VA30 + AgNPs } \\
(b)\end{array}$ & $\mathrm{FA}^{\ddagger}$ & $\begin{array}{l}\mathrm{P} 10 \\
(a) \\
\end{array}$ & $\begin{array}{c}\mathrm{P} 10+\text { AgNPs } \\
(b)\end{array}$ & $\mathrm{FA}^{*}$ & $\begin{array}{l}\text { RD5 } \\
\text { (a) }\end{array}$ & $\begin{array}{c}\mathrm{RD} 5+\text { AgNPs } \\
(b)\end{array}$ & $\mathrm{FA}^{\ddagger}$ \\
\hline S. enterica & $0^{\dagger}$ & $12 \pm 0.3$ & 3 & $0^{\dagger}$ & $12 \pm 1$ & 3 & $0^{\dagger}$ & $13 \pm 0.5$ & 3.69 \\
\hline E. coli & $0^{\dagger}$ & $13 \pm 0.4$ & 3.69 & $0^{\dagger}$ & $13 \pm 1.2$ & 3.69 & $0^{\dagger}$ & $13 \pm 0.2$ & 3.69 \\
\hline V. parahaemolyticus & $0^{\dagger}$ & $14 \pm 0.2$ & 4.44 & $0^{\dagger}$ & $14 \pm 1.5$ & 4.44 & $0^{\dagger}$ & $15 \pm 0.3$ & 5.25 \\
\hline Average & & & 3.71 & & & 3.71 & & & 4.21 \\
\hline B. anthracis & $15 \pm 0.5$ & $19 \pm 0.7$ & 0.6 & $0^{\dagger}$ & $15 \pm 0.5$ & 5.25 & $12 \pm 0.2$ & $16 \pm 0.7$ & 0.78 \\
\hline B. cereus & $17 \pm 0.2$ & $20 \pm 0.6$ & 0.38 & $8 \pm 0.3$ & $16 \pm 0.8$ & 3 & $13 \pm 0.3$ & $15 \pm 0.4$ & 0.33 \\
\hline S. aureus & $16 \pm 0.4$ & $20 \pm 0.2$ & 0.56 & $36 \pm 0.5$ & $40 \pm 0.8$ & 0.23 & $27 \pm 0.4$ & $29 \pm 0.6$ & 0.15 \\
\hline Average & & & 0.52 & & & 2.83 & & & 0.42 \\
\hline Pathogenic strain & & \multicolumn{3}{|c|}{$\mathrm{CHX}$} & \multicolumn{4}{|c|}{$\mathrm{CHX}+\mathrm{AgNPs}$} & $\mathrm{FA}^{\ddagger}$ \\
\hline C. albicans & & \multicolumn{3}{|c|}{$0^{\dagger}$} & \multicolumn{4}{|c|}{$13 \pm 0.5$} & 3.69 \\
\hline
\end{tabular}

Notes. ${ }^{\dagger}$ In the absence of bacterial growth inhibition zones, the disc's diameter $(6 \mathrm{~mm})$ was used to calculate the fold increases [13].

${ }^{\ddagger}$ Increase in fold area $(\mathrm{FA})=\left(b^{2}-a^{2}\right) / a^{2}$.

MY15: lincomycin $15 \mu \mathrm{g} /$ disk, OL15: oleandomycin $15 \mu \mathrm{g} /$ disk, VA30: vancomycin $30 \mu \mathrm{g} /$ disk, NV30: novobiocin $30 \mu \mathrm{g} /$ disk, P10: penicillin G $10 \mu \mathrm{g} / \mathrm{disk}$, and RD5: rifampicin $5 \mu \mathrm{g}$ /disk; CHX: cycloheximide, $10 \mu \mathrm{g} /$ disk; AgNPs: biosynthesised anisotropic silver nanoparticles, $30 \mu \mathrm{L}(100 \mathrm{ppm})$. 

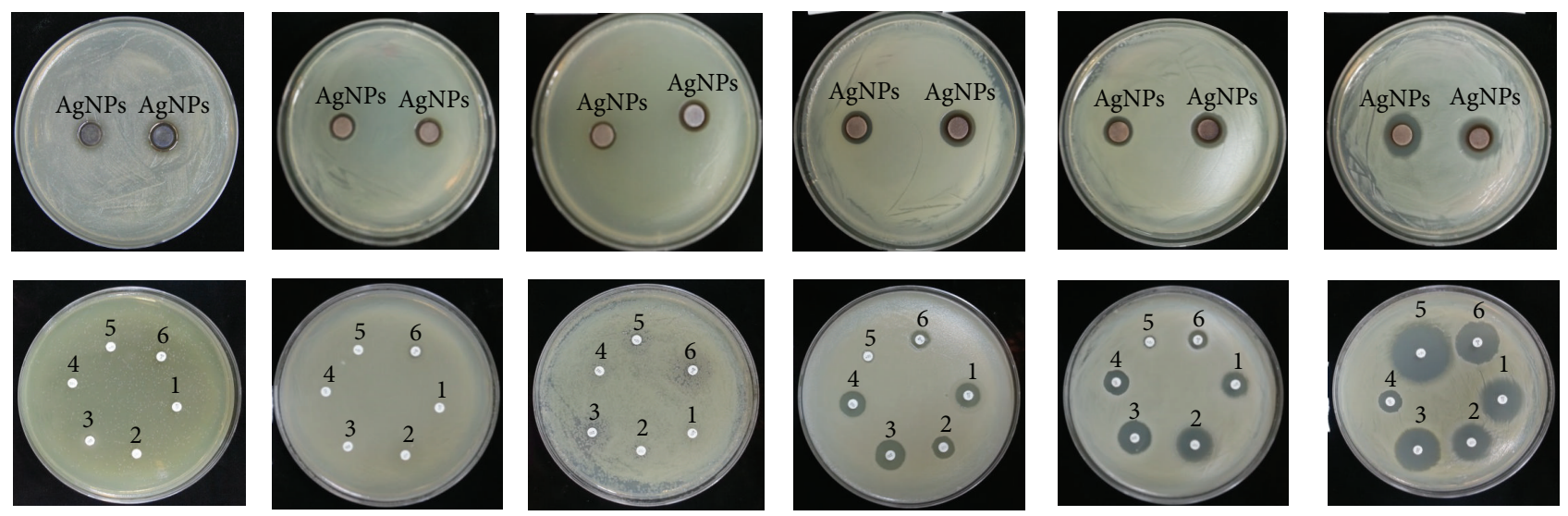

(a)

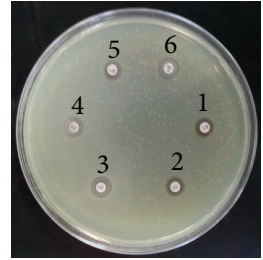

S. enterica

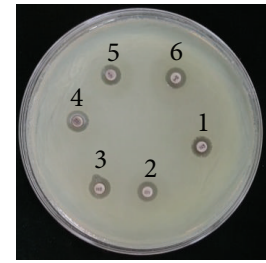

E. coli

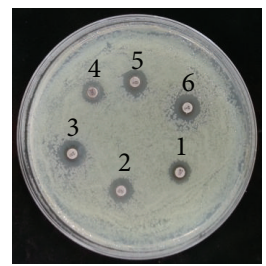

V. parahaemolyticus

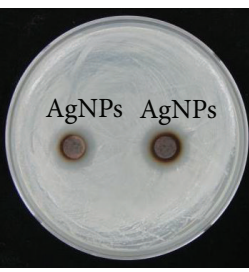

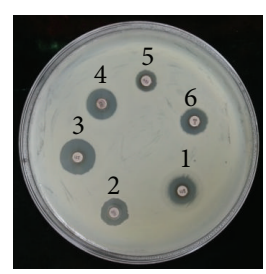

B. anthracis

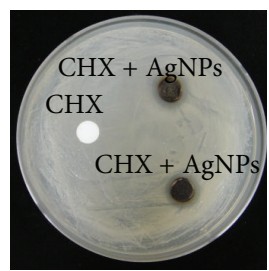

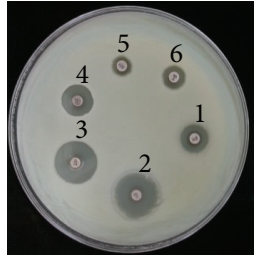

B. cereus

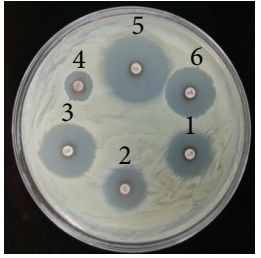

S. aureus

(b)

FIGURE 6: Zones of inhibition of partially purified silver nanoparticles pellet solution $30 \mu \mathrm{L}(100 \mathrm{ppm})$ and standard antibiotics as control, against Salmonella enterica, Escherichia coli, Vibrio parahaemolyticus, Bacillus anthracis, Bacillus cereus, and Staphylococcus aureus, respectively (a). Zones of inhibition of standard antibiotics disks with anisotropic silver nanoparticles against Salmonella enterica, Escherichia coli, Vibrio parahaemolyticus, Bacillus anthracis, Bacillus cereus, Staphylococcus aureus, and Candida albicans (b), respectively. Note. (1) Lincomycin (MY15) $15 \mu \mathrm{g} / \mathrm{disk}$, (2) oleandomycin (OL15) $15 \mu \mathrm{g} /$ disk, (3) novobiocin (NV30) $30 \mu \mathrm{g} / \mathrm{disk}$, (4) vancomycin (VA30) $30 \mu \mathrm{g} / \mathrm{disk}$, (5) penicillin G (P10) $10 \mu \mathrm{g} /$ disk, and (6) rifampicin (RD5) $5 \mu \mathrm{g} /$ disk; cycloheximide (CHX) $10 \mu \mathrm{g} /$ disk and anisotropic silver nanoparticles (AgNPs) $30 \mu \mathrm{L}(100 \mathrm{ppm})$.

silver nanoparticle solution was added and results in the formation of a zone of inhibition for S. enterica, E. coli, and $V$. parahaemolyticus. The average increase in sensitivity patter of antibiotics was calculated by average increase in fold area [35]. The average increase in fold area of the antibiotics against antibiotics resistant microorganisms was greatest for novobiocin, followed by rifampicin, lincomycin, and oleandomycin and then vancomycin and penicillin $G$. Similar results regarding increase in fold area have been observed against multidrug resistant E. coli [16]. The other pathogenic stains showed susceptibility to antibiotics disks alone, B. anthracis, B. cereus, and S. aureus, and showed increased susceptibility when the disks were combined with anisotropic silver nanoparticle solution, observed as increases in the diameter of the zone of inhibition for $B$. anthracis, B. cereus, and S. aureus. This clearly indicated the effect of biosynthesised anisotropic silver nanoparticles in enhancing the antimicrobial action of antibiotics.
The average increase in fold area against antibiotic sensitive microorganism, when anisotropic silver nanoparticles were added, was greatest for penicillin $\mathrm{G}$, followed by vancomycin, rifampicin, oleandomycin, novobiocin, and lincomycin. In addition, in case of cycloheximide, the similar pattern of action was found against $C$. albicans. On the addition of silver nanoparticles with cycloheximide, the activity of cycloheximide increases. The study has been done in duplicate and average results were interpreted in Table 2 . The present study shows results similar to those of a line of studies that presented increased efficacies of commercial antibiotics against pathogenic microorganisms $[13,16,28]$. It indicates that biosynthesised anisotropic silver nanoparticles show antimicrobial activity against pathogenic microorganisms. Moreover, the nanoparticles were shown to enhance the effect of commercial antibiotics against multidrug resistant bacteria. Thus, the biosynthesised anisotropic silver nanoparticles are ready for applications in the clinical field against 
pathogenic microorganisms, although the exact mechanism of their action remains to be elucidated.

\section{Conclusions}

The most important advantage of this approach includes the possibility to obtain anisotropic silver nanoparticles of nanobar, pentagon, spherical, icosahedron, hexagonal, truncated triangle, and triangular shapes by Bhargavaea indica DC1. The anisotropic silver nanoparticles were synthesized by eco-friendly and economical manner and eliminating the hazardous effects of physiochemical synthesis. Moreover, the particles were synthesized extracellularly, without any extra reducing and capping agent, thus excluding the extra step in physiochemical synthesis. Furthermore, the methodology has greater advantage of easy bulk synthesis which can be exploited for large scale industrial scale-up production due to easy downstream processing.

The outcomes of study also indicated that the biosynthesised anisotropic silver nanoparticles had inherent antimicrobial activity and also enhanced the antimicrobial activity of commercial antibiotics against pathogenic microorganisms. The biosynthesized silver nanoparticles may have potential to be used as multifunctional nanoparticles for different applications on medical, clinical, and biological background such as targeting, diagnosis, drug delivery, and photoimaging. Further studies in order to understand the mechanism of action, surface engineering, specific targeting, and conjugation may be useful to develop novel nanoparticles for different applications.

\section{Conflict of Interests}

The authors declare that there is no conflict of interests regarding the publication of this paper.

\section{Acknowledgments}

This research was supported by the Korea Institute of Planning and Evaluation for Technology in Food, Agriculture, Forestry and Fisheries (KIPET no. 313038-03-2-SB010) and by a Next-Generation BioGreen 21 Program (SSAC, Grant no.: PJ009529032014) of the Republic of Korea.

\section{References}

[1] L. Zhang, F. X. Gu, J. M. Chan, A. Z. Wang, R. S. Langer, and O. C. Farokhzad, "Nanoparticles in medicine: therapeutic applications and developments," Clinical Pharmacology and Therapeutics, vol. 83, no. 5, pp. 761-769, 2008.

[2] N. Kulkarni and U. Muddapur, "Biosynthesis of metal nanoparticles: a review," Journal of Nanotechnology, vol. 2014, Article ID 510246, 8 pages, 2014.

[3] M. Kowshik, S. Ashtaputre, S. Kharrazi et al., "Extracellular synthesis of silver nanoparticles by a silver-tolerant yeast strain MKY3," Nanotechnology, vol. 14, no. 1, p. 95, 2003.

[4] N. Jain, A. Bhargava, S. Majumdar, J. C. Tarafdar, and J. Panwar, "Extracellular biosynthesis and characterization of silver nanoparticles using Aspergillus flavus NJP08: a mechanism perspective," Nanoscale, vol. 3, no. 2, pp. 635-641, 2011.
[5] J. J. Antony, P. Sivalingam, D. Siva et al., "Comparative evaluation of antibacterial activity of silver nanoparticles synthesized using Rhizophora apiculata and glucose," Colloids and Surfaces B: Biointerfaces, vol. 88, no. 1, pp. 134-140, 2011.

[6] P. Sivalingam, J. J. Antony, D. Siva, S. Achiraman, and K. Anbarasu, "Mangrove Streptomyces sp. BDUKAS10 as nanofactory for fabrication of bactericidal silver nanoparticles," Colloids and Surfaces B: Biointerfaces, vol. 98, pp. 12-17, 2012.

[7] M. A. Dar, A. Ingle, and M. Rai, "Enhanced antimicrobial activity of silver nanoparticles synthesized by Cryphonectria sp. evaluated singly and in combination with antibiotics," Nanomedicine: Nanotechnology, Biology, and Medicine, vol. 9, no. 1, pp. 105-110, 2013.

[8] A. R. Shahverdi, A. Fakhimi, H. R. Shahverdi, and S. Minaian, "Synthesis and effect of silver nanoparticles on the antibacterial activity of different antibiotics against Staphylococcus aureus and Escherichia coli," Nanomedicine: Nanotechnology, Biology, and Medicine, vol. 3, no. 2, pp. 168-171, 2007.

[9] S. Pal, Y. K. Tak, and J. M. Song, "Does the antibacterial activity of silver nanoparticles depend on the shape of the nanoparticle? A study of the gram-negative bacterium Escherichia coli," Applied and Environmental Microbiology, vol. 73, no. 6, pp. 17121720, 2007.

[10] S. Prabhu and E. K. Poulose, "Silver nanoparticles: mechanism of antimicrobial action, synthesis, medical applications, and toxicity effects," International Nano Letters, vol. 2, no. 1, article 32, 2012.

[11] S. Gurunathan, K. Kalishwaralal, R. Vaidyanathan et al., "Biosynthesis, purification and characterization of silver nanoparticles using Escherichia coli," Colloids and Surfaces B: Biointerfaces, vol. 74, no. 1, pp. 328-335, 2009.

[12] N. Sanvicens and M. P. Marco, "Multifunctional nanoparticles-properties and prospects for their use in human medicine," Trends in Biotechnology, vol. 26, no. 8, pp. 425-433, 2008.

[13] S. S. Birla, V. V. Tiwari, A. K. Gade, A. P. Ingle, A. P. Yadav, and M. K. Rai, "Fabrication of silver nanoparticles by Phoma glomerata and its combined effect against Escherichia coli, Pseudomonas aeruginosa and Staphylococcus aureus," Letters in Applied Microbiology, vol. 48, no. 2, pp. 173-179, 2009.

[14] M. Tréguer-Delapierre, J. Majimel, S. Mornet, E. Duguet, and S. Ravaine, "Synthesis of non-spherical gold nanoparticles," Gold Bulletin, vol. 41, no. 2, pp. 195-207, 2008.

[15] S. S. Shankar, A. Ahmad, R. Pasricha, and M. Sastry, "Bioreduction of chloroaurate ions by geranium leaves and its endophytic fungus yields gold nanoparticles of different shapes," Journal of Materials Chemistry, vol. 13, no. 7, pp. 1822-1826, 2003.

[16] S. Priyadarshini, V. Gopinath, N. Meera Priyadharsshini, D. MubarakAli, and P. Velusamy, "Synthesis of anisotropic silver nanoparticles using novel strain, Bacillus flexus and its biomedical application," Colloids and Surfaces B: Biointerfaces, vol. 102, pp. 232-237, 2013.

[17] A. M. Goswami and S. Ghosh, "Biological synthesis of colloidal gold nanoprisms using Penicillium citrinum MTCC9999," Journal of Biomaterials and Nanobiotechnology, vol. 4, no. 2, pp. 2027, 2013.

[18] H. H. Lara, N. V. Ayala-Núñez, L. C. I. Turrent, and C. R. Padilla, "Bactericidal effect of silver nanoparticles against multidrug-resistant bacteria," World Journal of Microbiology and Biotechnology, vol. 26, no. 4, pp. 615-621, 2010. 
[19] M. K. Rai, S. D. Deshmukh, A. P. Ingle, and A. K. Gade, "Silver nanoparticles: the powerful nanoweapon against multidrugresistant bacteria," Journal of Applied Microbiology, vol. 112, no. 5, pp. 841-852, 2012.

[20] W. G. Weisburg, S. M. Barns, D. A. Pelletier, and D. J. Lane, "16S ribosomal DNA amplification for phylogenetic study," Journal of Bacteriology, vol. 173, no. 2, pp. 697-703, 1991.

[21] K. K. Kim, M. K. Kim, J. H. Lim, H. Y. Park, and S.T. Lee, "Transfer of Chryseobacterium meningosepticum and Chryseobacterium miricola to Elizabethkingia gen. nov. as Elizabethkingia meningoseptica comb. nov. and Elizabethkingia miricola comb. nov," International Journal of Systematic and Evolutionary Microbiology, vol. 55, no. 3, pp. 1287-1293, 2005.

[22] L. Wang, Q. M. Liu, B. H. Sung et al., "Bioconversion of ginsenosides $R b_{1}, R b_{2}, R c$ and $R d$ by novel beta-glucosidase hydrolyzing outer 3-O glycoside from Sphingomonas sp. 2F2: cloning, expression, and enzyme characterization," Journal of Biotechnology, vol. 156, no. 2, pp. 125-133, 2011.

[23] R. T. Vendan, Y. J. Yu, S. H. Lee, and Y. H. Rhee, "Diversity of endophytic bacteria in ginseng and their potential for plant growth promotion," Journal of Microbiology, vol. 48, no. 5, pp. 559-565, 2010.

[24] K. M. Cho, S. Y. Hong, S. M. Lee et al., "Endophytic bacterial communities in ginseng and their antifungal activity against pathogens," Microbial Ecology, vol. 54, no. 2, pp. 341-351, 2007.

[25] O.-S. Kim, Y.-J. Cho, K. Lee et al., "Introducing EzTaxon-e: a prokaryotic 16s rRNA gene sequence database with phylotypes that represent uncultured species," International Journal of Systematic and Evolutionary Microbiology, vol. 62, part 3, pp. 716-721, 2012.

[26] P. Verma, C. N. Seong, P. K. Pandey et al., "Bhargavaea indica sp. nov., a member of the phylum Firmicutes, isolated from Arabian Sea sediment," Journal of Microbiology, vol. 51, no. 1, pp. 36-42, 2013.

[27] A. Maciollek and H. Ritter, "One pot synthesis of silver nanoparticles using a cyclodextrin containing polymer as reductant and stabilizer," Beilstein Journal of Nanotechnology, vol. 5, no. 1, pp. 380-385, 2014.

[28] S. Z. H. Naqvi, U. Kiran, M. I. Ali et al., "Combined efficacy of biologically synthesized silver nanoparticles and different antibiotics against multidrug-resistant bacteria," International Journal of Nanomedicine, vol. 8, pp. 3187-3195, 2013.

[29] V. Sharma, K. Park, and M. Srinivasarao, "Shape separation of gold nanorods using centrifugation," Proceedings of the National Academy of Sciences of the United States of America, vol. 106, no. 13, pp. 4981-4985, 2009.

[30] R. Ramanathan, A. P. O'Mullane, R. Y. Parikh, P. M. Smooker, S. K. Bhargava, and V. Bansal, "Bacterial kinetics-controlled shape-directed biosynthesis of silver nanoplates using Morganella psychrotolerans," Langmuir, vol. 27, no. 2, pp. 714-719, 2011.

[31] S. K. Das, A. R. Das, and A. K. Guha, "Microbial synthesis of multishaped gold nanostructures," Small, vol. 6, no. 9, pp. 10121021, 2010.

[32] H. Schneidewind, T. Schüler, K. K. Strelau et al., "The morphology of silver nanoparticles prepared by enzyme-induced reduction," Beilstein Journal of Nanotechnology, vol. 3, no. 1, pp. 404-414, 2012.

[33] S. Sadhasivam, P. Shanmugam, and K. Yun, "Biosynthesis of silver nanoparticles by Streptomyces hygroscopicus and antimicrobial activity against medically important pathogenic microorganisms," Colloids and Surfaces B: Biointerfaces, vol. 81, no. 1, pp. 358-362, 2010.

[34] A. R. Poda, A. J. Bednar, A. J. Kennedy et al., "Characterization of silver nanoparticles using flow-field flow fractionation interfaced to inductively coupled plasma mass spectrometry," Journal of Chromatography A, vol. 1218, no. 27, pp. 4219-4225, 2011.

[35] J. R. Morones, J. L. Elechiguerra, A. Camacho et al., "The bactericidal effect of silver nanoparticles," Nanotechnology, vol. 16, no. 10, pp. 2346-2353, 2005. 

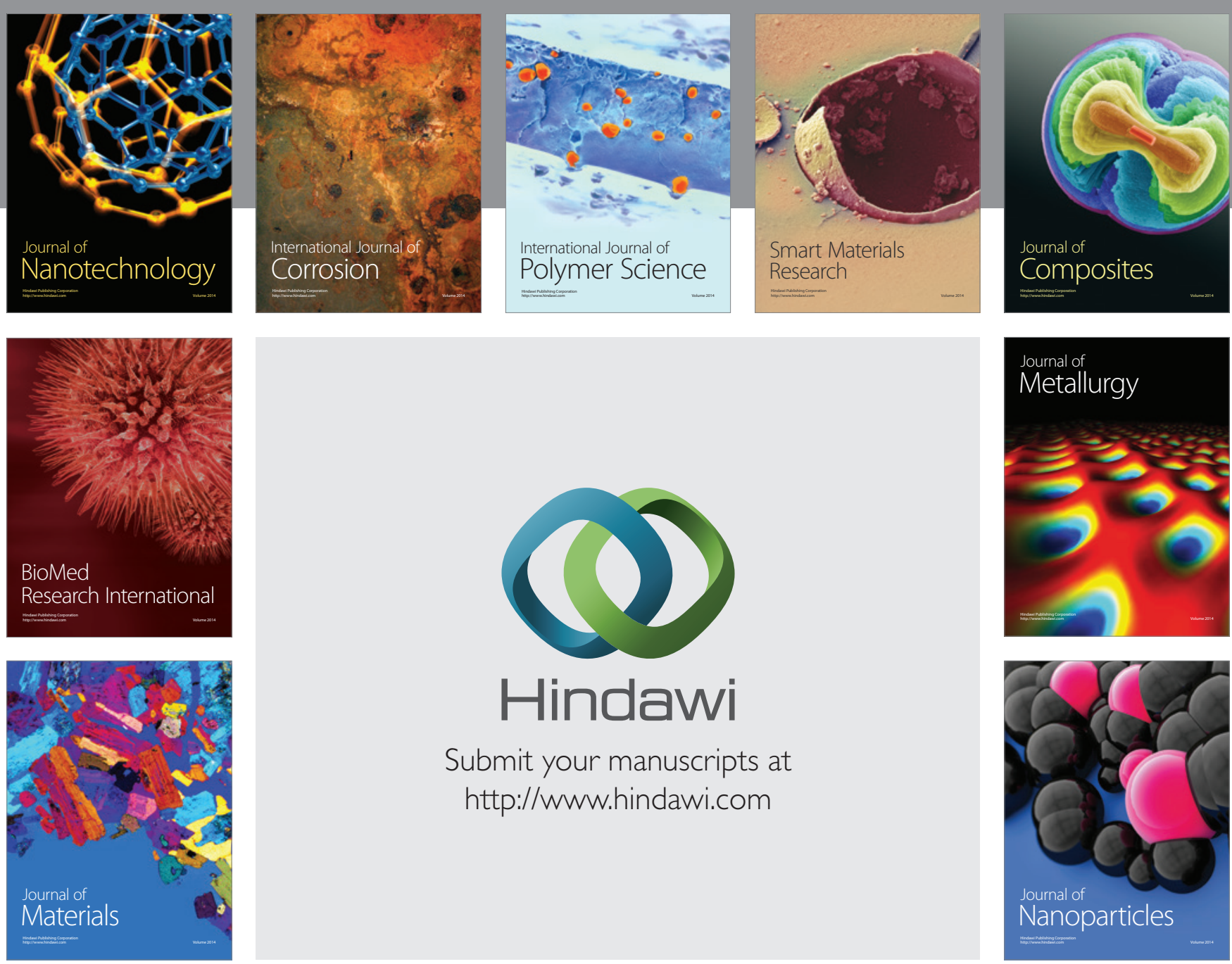

Submit your manuscripts at http://www.hindawi.com
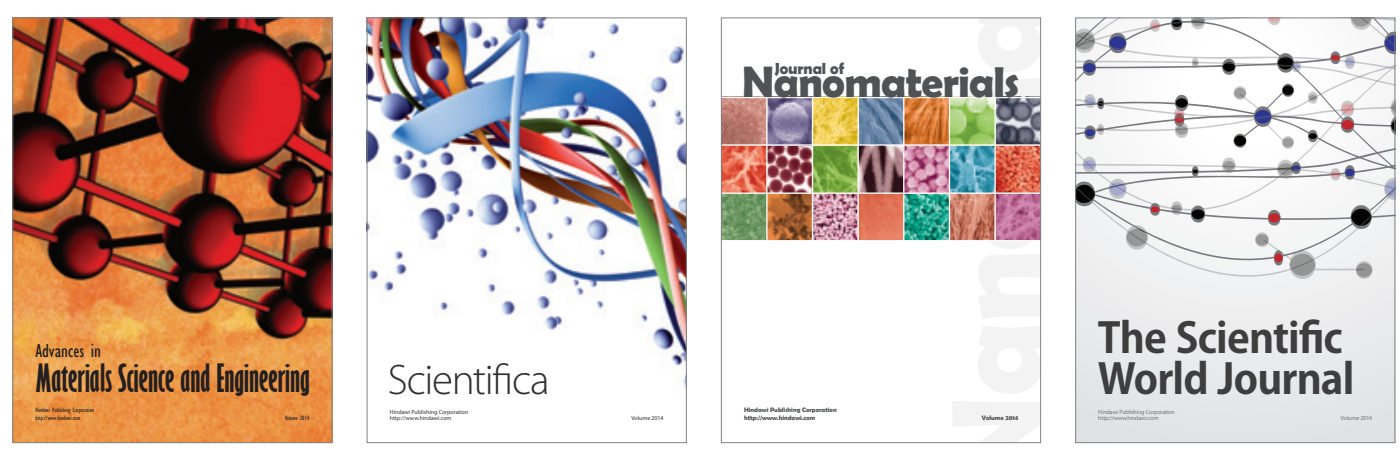

\section{The Scientific World Journal}
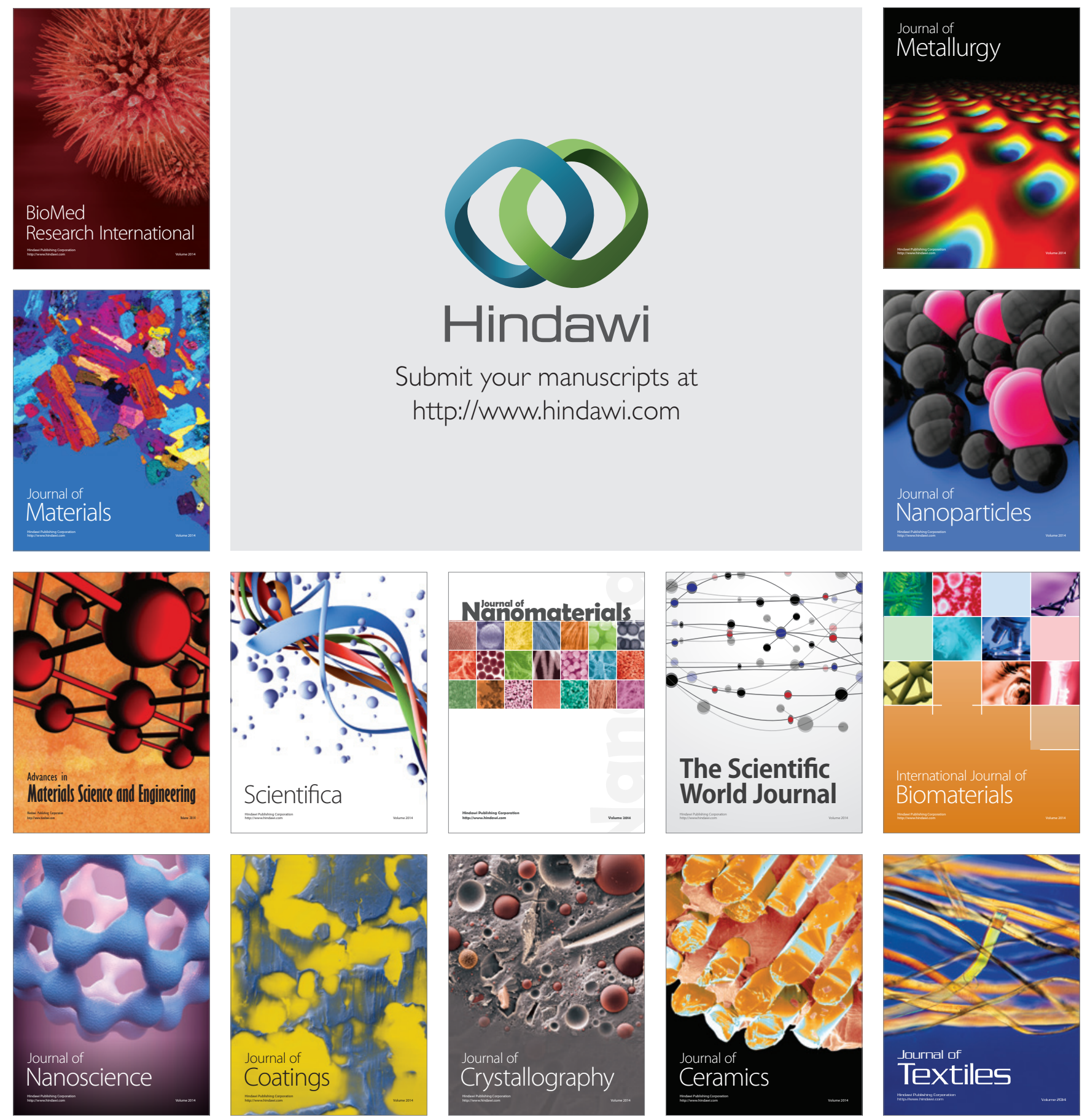